\title{
Chemistry and Structure of AOT Surfactants on Mica Investigated with Cryogenic Electron Microscopy and Molecular Dynamics
}

DANIEL M. LONG ${ }^{1}$, GUANGPING XU² ${ }^{2}$ HONGKYU YOON ${ }^{3}$, JEFFERY A. GREATHOUSE ${ }^{4}$, KATHERINE L. JUNGJOHANN $^{5}$

Center for Integrated Nanotechnologies, Sandia National

Laboratories, NM, USA

11mlong@sandia.gov, ${ }^{5}$ kljungj@sandia.gov

Geochemistry Department, Sandia National Laboratories, NM, USA

${ }^{2}$ gxu@sandia.gov, ${ }^{4}$ jagreat@sandia.gov

Geoscience Research \& Applications, Sandia National

Laboratories, NM, USA

3hyoon@sandia.gov

Anionic complex fluids have been shown to, unexpectedly, bind to anionic surfaces, which can be utilized to manipulate surface wettability. Understanding the interaction between complex fluids and minerals at the solid/liquid interface is key to improved processes related to subsurface energy and water applications. With mica and the anionic surfactant AOT as our model system, we identify the effect of the charge-balancing cation on the partitioning of the surfactant between the adsorbed and diffuse layers.

Recent advances in cryogenic electron microscopy (cryoEM) preparation have made it possible to use scanning transmission electron microscopy (STEM) to investigate the intact solid/liquid interfaces and electron-beam-sensitive materials. Here we utilize cryo-EM to investigate nanoscale liquid-mineral interfacial interactions. These nanoscale results are integrated with results from molecular dynamics simulations and macroscopic characterization from Raman spectra and contact angle measurements to understand the mechanism of interfacial interactions between mica and surfactant. We present the microstructure of the mica/AOT interface coupled with local chemistry from cryo-EM investigations which utilize cryo-FIB preparation, cryotransfer, and cryo-STEM. Details will be presented on the AOT interfacial structure, including high-resolution surfactant structure and surfactant chemistry at the interface.

SNL is managed and operated by NTESS under DOE NNSA contract DE-NA0003525. 